\title{
The design and utility of polymer-stabilized iron-oxide nanoparticles for nanomedicine applications
}

\author{
Cyrille Boyer $^{1 *}$, Michael R. Whittaker ${ }^{1}$, Volga Bulmus ${ }^{2}$, Jingquan Liu ${ }^{1}$ and Thomas P. Davis ${ }^{1 *}$ \\ University of New South Wales, Australia
}

Over the past decade, the synthesis of superparamagnetic nanoparticles, especially iron-oxide nanoparticles (IONPs), has been researched intensively for many high-technology applications, including enhanced storage media, biosensing and medical applications. In medicine, IONPs are used as contrast agents in magnetic resonance imaging and in hyperthermia therapy, and can also be exploited in drug or gene delivery as they are relatively non-toxic. However, their usage in vivo is limited by their agglomeration in biological fluids, induced by their high surface energies and tendency to adsorb proteins. The addition of a polymer layer to the surface of IONPs can stabilize these nanoparticles, giving well-dispersed individual nanoparticles in biological fluids for in vitro and in vivo applications, thereby increasing the blood circulation half-life. Moreover, the polymer layer can endow the IONPs with functionality, providing a scaffold for the encapsulation or attachment of therapeutic agents (drugs or genes), targeting agents and permeation enhancers. This review examines recent advancements in the use of IONPs in medicine, a field that has been particularly active in the last few years.

\begin{abstract}
ron-oxide nanoparticles (IONPs) represent a significant class of inorganic nanomaterial that is contributing to the current revolution in nanomedicine [1-2]. Their unique physical properties, including high surface area to volume ratios and superparamagnetism, confer useful attributes for medical applications such as magnetic resonance imaging (MRI), drug and gene delivery, tissue engineering and bioseparation [3].

Two distinct classes of superparamagnetic IONP-based materials are currently used for medical applications: superparamagnetic iron-oxide (SPIO) nanoparticles with a mean particle diameter of 50-100 nm, and ultra-small superparamagnetic iron-oxide (USPIO) nanoparticles with a size below $50 \mathrm{~nm}$. These two classes of IONPs have been studied widely for medical applications, particularly as the next (potential) generation of MRI contrast agents. They are also seen as potential vectors for drug and gene delivery. The biodistribution of these nanoparticles can be altered by the application of an external magnetic field; they also have potential applications in hyperthermia therapy as some magnetic particles can heat up under the influence of a localized high-frequency magnetic field.

The intent of this review is to present recent advances in the synthesis of IONPs and their subsequent stabilization in biological fluids using polymers, focusing on the current strategies used to graft polymers onto IONPs surfaces (see Figure 1) and the different types of polymers used. The additional properties conferred by the polymers, such as targeting, effects on biodistribution and pharmacokinetics, are also discussed, and the main applications of IONPs are reviewed.
\end{abstract}

\section{Synthesis and properties of iron-oxide nanoparticles}

SPIO and USPIO nanoparticles are the most extensively studied magnetic nanoparticles for biomedical applications as they are both biocompatible and easy to synthesize. Both are composed of ferrite nanocrystallites of magnetite $\left(\mathrm{Fe}_{3} \mathrm{O}_{4}\right)$ or maghemite $\left(\mathrm{Fe}_{2} \mathrm{O}_{3}, \gamma\right)$. Over the last ten years, there has been an explosion of interest in these materials and this has

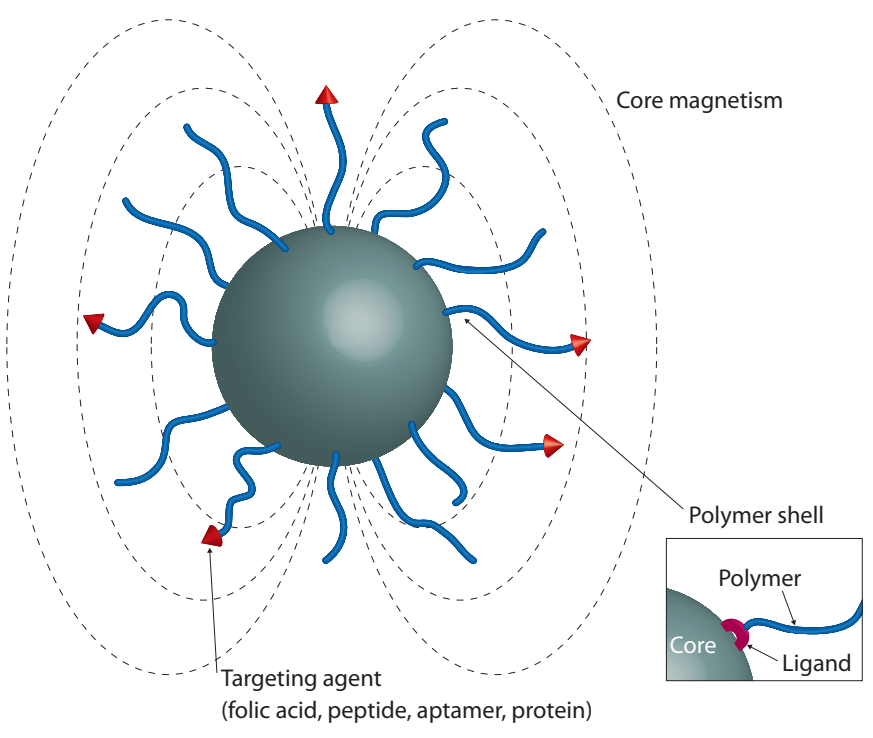

Figure 1. Functional iron-oxide nanoparticles. Functional polymers are grafted onto an inorganic core of magnetite $\left(\mathrm{Fe}_{3} \mathrm{O}_{4}\right)$ or maghemite $\left(\mathrm{Fe}_{2} \mathrm{O}_{3}\right)$ through an anchoring group, such as an amine, carboxylic acid or phosphonic acid group. The polymer shell improves the stability of IONPs in solution, and also allows the encapsulation of a therapeutic agent.

been reflected in a large number of recent publications describing the synthesis and modification of hybrid IONPs. In this review, we focus on the polymer modification of the nanoparticles, and provide only minimal information on the inorganic synthesis of IONPs. For more detailed information on IONP synthesis, readers are referred to other reviews [3,4]. 
A concise description of IONP synthesis follows. The coprecipitation process is the simplest and most widely employed chemical route for the synthesis of IONPs. Briefly, IONPs are prepared by aging a stoichiometric mixture of ferrous and ferric salts in aqueous media under basic conditions to yield magnetite in the absence of oxygen. However, this oxidation state is unstable and can quickly transform to maghemite in air, or under acidic conditions in the absence of oxygen. The main advantage of this approach is that it produces a large amount of material, with control over particle size $(2-20 \mathrm{~nm})$ and shape afforded by adjusting $\mathrm{pH}$, ionic strength and the concentration of the growth solution. In addition, the nanoparticles can be functionalized in situ using additives such as organic compounds (e.g. sodium citric) or polymers (e.g. dextran, polyvinyl alcohol). The magnetic properties of the nanoparticles can also be tailored. For instance, the saturation magnetization varies with the concentration of salt $(\mathrm{NaCl})$ used in the synthesis (63-71 emu/g for magnetite).

Another method, high-temperature decomposition of organo-metallic precursors $\left(\right.$ e.g. $\left.\mathrm{Fe}(\mathrm{CO})_{5}\right)$ in organic solvents, offers improved control over the size and shape of IONPs [5]. The IONP size (3-19nm) can be tuned by the choice of precursor and temperature. Shape control (i.e. yielding spherical particles) is also enhanced using the decomposition process. The IONPs produced by decomposition are coated with hydrophobic compounds to facilitate stabilization in organic solvents, however this reduces their stability in aqueous biological environments. The magnetic properties of IONPs can be tuned by the incorporation of other metals, such as cobalt, nickel and manganese, into the material [6].

Another way to synthesize IONPs is by flame spray pyrolysis [7], yielding a wide range of IONPs, such as magnetite, maghemite and wustite $(\mathrm{FeO})$ particles. The iron oxidation state can be controlled by varying the fuel-to-air ratio during combustion, as well as by varying the valence state of the iron precursor $\left(\mathrm{Fe}(\mathrm{CO})_{5}, \mathrm{Fe}\left(\mathrm{NO}_{3}\right)_{3}\right.$, etc.). Flame spray pyrolysis presents useful advantages: it can produce fine particles $(6-50 \mathrm{~nm})$ on a large scale and can be easily scaled up using industrial plant (several grams per hour), it limits the presence of impurities on the nanoparticles, and it can be extended to produce different nanoparticles $\left(\mathrm{TiO}_{2}, \mathrm{ZrO}_{2}\right.$, silica, etc.) as well as hybrid particles (e.g. silica-IONPs) [8].

At low temperature, IONPs self-organize in solution; all magnetic spins align resulting in ferromagnetism. When the temperature is sufficiently high, thermal energy can invert the direction of magnetic spin, disrupting spin alignment. Above this temperature (the blocking temperature), the nanoparticle ensemble loses its magnetic properties. The application of an external magnetic field induces the spontaneous reorganization of magnetic spin directions and re-magnetizes the IONPs. If the magnetic field is removed, the particles lose their spin alignment, liberating heat. Ferromagnetism is essential for the synthesis of stable colloidal IONP suspensions, as the non-alignment of spin limits the inter-particle attraction that would otherwise occur. Superparamagnetism is exhibited by very small particles. The saturation magnetization or magnetization (i.e. $M_{s}$, units of emu/g), defined by the alignment of all magnetic spins in a sample, decreases with IONP size [9]. The magnetic spins of atoms close to the surface are less well organized than for atoms in the bulk of the particle near the core (Figure 2). This phenomenon is referred to as 'spin canting'. In addition, high crystallinity results in higher saturation magnetization [9]. To improve $M_{\mathrm{s}}$, the addition of dopants, such as manganese, has been reported [6]. Magnetite (92 emu/g) has a higher $M_{\mathrm{s}}$ value than maghemite $(78 \mathrm{emu} / \mathrm{g}$ ) for a similar particle size [10].

An important property of IONPs, or magnetic nanoparticles in general, is their ability to accelerate the MRI relaxation processes of surrounding water protons, resulting in enhanced MRI contrast. Briefly, in MRI, an external magnetic field is applied to the IONPs, resulting in the alignment of nanoparticle magnetic spin, inducing a magnetic dipole moment. The protons of water molecules within the magnetic field of the nanoparticles have a magnetic relaxation time significantly different to that of water molecules outside the IONPs' magnetic field. An accumulation of IONPs at a specific site therefore produces a significant enhancement in contrast in an MR image.

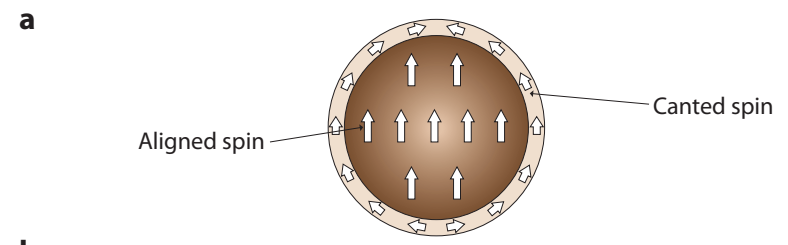

b
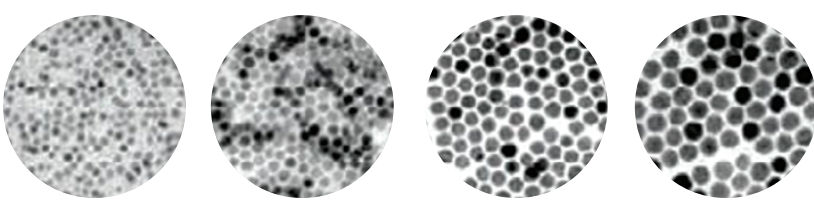

Particle size

$4 \mathrm{~nm}$ $6 \mathrm{~nm}$ $9 \mathrm{~nm}$ $50 \mathrm{~nm}$

\section{c}
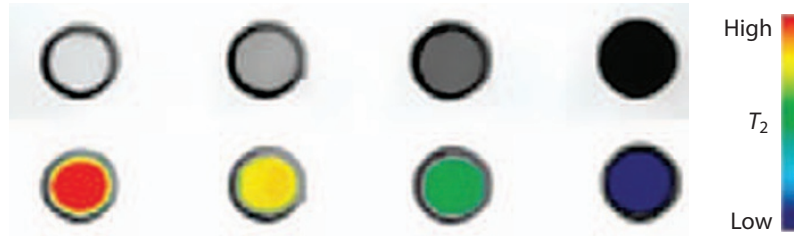

Figure 2. Evolution of magnetic properties with nanoparticle size. (a) Illustration of spin canting, the disorganization of spin close to the surface due to a lack of crystallinity organization. (b) Transmission electronic microscopy images of various IONPS synthesized through the thermal decomposition of Fe(acetylacetonate) ${ }_{3}$ in benzyl ether. (c) Size-dependent $T_{2}$-weighted MR images of IONPS in aqueous solution at 1.5T. Adapted from Ref. 9 (๔ 2005 ACS).

Magnetism can also be exploited to exert control over the biodistribution of IONPs. Several successful examples of magnetic targeting have been reported in the literature, as discussed later.

\section{Modification of IONPs}

'Naked' IONPs are not stable in water (at neutral $\mathrm{pH}$ ) or in physiological fluids, tending to agglomerate and precipitate quickly. Either steric or electrostatic stabilization of the IONPs is required to ensure stable aqueous dispersions. A range of different functionalities and approaches can be used to attain colloidal stability.

\section{Anchoring functionality}

The presence of hydroxyl groups, such as $\mathrm{Fe}-\mathrm{OH}$, on the IONP surfaces provides a versatile synthetic handle allowing attachment of different functionalities. A range of chemistries can be used to stabilize metal nanoparticles, exploiting electrostatic, hydrophobic, chelating and covalent interactions (Figure 3).

One of the most commonly used surface modification techniques is the attachment of alkoxysilane compounds. Silane can be covalently attached onto IONP surfaces by reaction of the surface $\mathrm{Fe}-\mathrm{OH}$ group with the $\mathrm{Si}-\mathrm{OCH}_{3}$ moiety [11]. Further cross-linking events produce a thin inorganic silica layer around the particles [12]. However, during this cross-linking process, the irreversible formation of aggregates can be observed, limiting the use of the resultant IONPs. The functionalization of IONPs using silane chemistry is versatile, involving the use of many functional silanes available commercially, such as alcohol, amine and thiol, which are useful for further biofunctionalization using small biocompounds [12] and carbohydrates [13].

Another important and widely employed functionality for the modification of IONP surfaces is the carboxylic acid group, which can interact with the surface of IONPs by coordination processes. The $\mathrm{COOH}$ group has been employed for IONP synthesis in organic solvents (oleic acid). Citric acid has been used commercially for the 


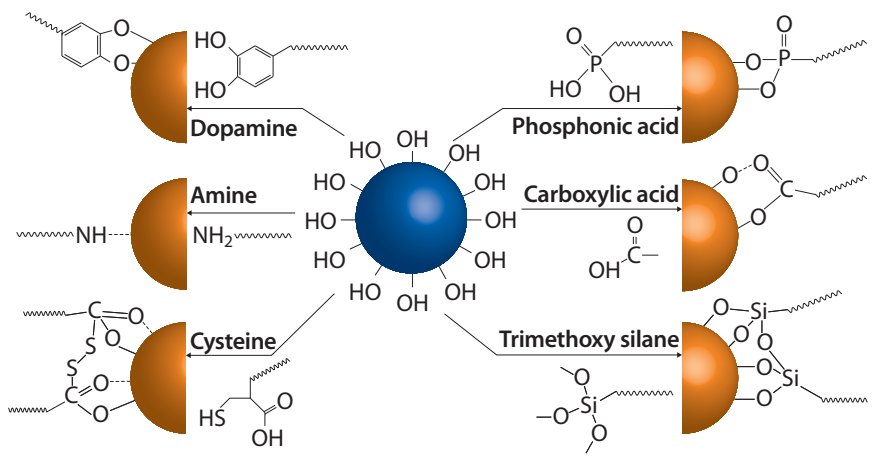

Figure 3. Groups that can be used to anchor polymers on IONP surfaces.

stabilization of IONPs, such as in the MRI contrast agent VSOP C184. However, the $-\mathrm{COOH} / \mathrm{IONP}$ coordination bond is labile and can be broken easily by increasing temperature or by exchange with another carboxylic acid compound.

Phosphonic acid also shows a strong affinity for IONP surfaces through the formation of Fe-O-P- bonds [14]. These bonds are more stable than the carboxylic acid bond and have shown stability for several weeks at neutral $\mathrm{pH}$. Phosphonic acid also shows a higher grafting density than the carboxylic acid group [14].

Finally, dopamine can coordinate to the IONP surfaces as a result of improved orbital overlap of the five-member ring [15]. This approach has been widely used to attach a range of biologically important molecules, such as peptides and amino acids, to IONPs [16]. However, problems with the stability of this bond in water and biological fluids have been reported by Carpenter et al. after long exposure periods [17].

Phosphonic acid and dopamine groups appear to improve IONP stability over a larger range of $\mathrm{pH}$ and temperature compared with carboxylic acid groups.

\section{Stabilization of IONPs using polymer chains}

For many applications in medicine, a polymer coating on the IONPs is preferred over simple functionalization with small organic compounds. A polymer coating provides colloidal stability in water through steric stabilization, and can provide surface functionality allowing the possibility of designing hybrid particles with capacity for multimodal tracking, targeting, delivery and stimulated release of therapeutic agents such as peptides, proteins and DNA/RNA.

Grafting 'onto' versus 'from'. Polymer attachment to the IONP surface can be achieved via two alternative approaches: grafting 'onto' and 'from'. In the case of grafting 'from', an initiator is fixed to the surface of the IONPs and the polymer is grown from the surface, while in the grafting 'onto' approach, a functional, pre-formed polymer is grafted onto IONPs in situ. Each of these approaches has advantages and disadvantages. It is well known that grafting 'from' yields a higher grafting density than the grafting 'onto' approach. However, grafting 'onto' allows control of polymer architecture and functionality, and is therefore more versatile than the grafting 'from' method. In addition, grafting 'from' can present difficulties in maintaining the integrity of the hybrid nanoparticles in organic solvents. However, the use of living radical polymerization, with a carefully designed protocol, can facilitate the modification of IONPs using the grafting 'from' approach. For example, Hatton et al. [18] grafted several polymers from IONPs coated with an atom transfer radical polymerization (ATRP) initiator yielding polymer/IONP hybrids. Ring-opening polymerization (ROP) has also been employed to obtain IONPs coated with linear biodegradable poly(esters) [19] or hyper-branched polymers [20]. Finally, a grafting 'through' method has been described using IONPs coated with methacrylic bonds to yield well-dispersed particle solutions [21].
Stabilization of IONPs using monofunctional polymer chains via exchange chemistry. The attachment of polymer chains onto IONPs can be achieved using polymers with functionality capable of binding to the IONP surface. For example, the Centre for Advanced Macromolecular Design has developed an anti-fouling polymer-coated maghemite made by grafting 'onto' of phosphonic acid-terminated poly(oligoethylene glycolacrylate) (poly(OEG-A)), yielding hybrid organic/inorganic nanoparticles that form stable dispersions in both water and fetal bovine serum [22].

Stayton, Hoffman and co-workers [23] used a terminal carboxylic acid group on telechelic poly $(N$-isopropylacrylamide) to modify IONPs. In a similar approach, Gao et al. [24] used cysteine-terminated polyethylene glycol (PEG) anchored to the surface of IONPs. The presence of both carboxylic acid and thiol groups allows the anchoring of the polymers and the simultaneous cross-linking of these polymers around the nanoparticles. This was achieved though oxidation of the thiol groups to disulfide crosslinking groups. The great advantage of this route is that it yields nanoparticles that are stable in biological fluid and addresses the problem of the labile and unstable nature of the interaction between carboxylic acid and IONPs.

Kohler et al. [25] proposed the use of a telechelic polymer having a terminal trimethylsilyl group able to react onto IONPs and a peptide able to confer targeting properties coupled to the other terminus of the polymer. De Palma [12] showed that the silane approach allows the preparation of stable hybrid nanoparticles over a large range of $\mathrm{pH}$, resulting from strong covalent $\mathrm{FeO}-\mathrm{Si}$ - bonds.

Dopamine has also been used as the ligand to attach polymers onto IONPs. Dopamine-terminated PEG was fixed onto IONPs to stabilize these particles in biological fluids. The polymer also had a terminal biotin group. The presence of biotin was exploited for the attachment of antibodies using the well-known biotin-neutravidin recognition reaction [26].

The recent development of high-yield 'click' chemistries, such as the azide-alkyne reaction [27], now provides alternative routes to stabilized IONPs. For example, Turro et al. [28] described the stabilization of $\mathrm{Fe}_{2} \mathrm{O}_{3}$ nanoparticles using alkyne-terminated organophosphate or carboxylic acid groups to exchange with oleic acid on the $\mathrm{Fe}_{2} \mathrm{O}_{3}$ surface. The IONPs were subsequently covalently attached to poly(tert-butyl acrylate) via click reactions using $\mathrm{CuSO}_{4}$.

Stabilization of IONPs using functional diblock polymers. The attachment of polymer chains to IONPs can also be achieved via multiple interactions between the polymer chains and the particle surface. This multiple attachment strategy should enhance the stability of the resultant hybrid nanoparticles, and can be achieved through the use of block or random copolymers. The insertion of several functional groups along the copolymer backbone increases the number of possible anchoring points on the IONP surface. The control of polymer architecture is a crucial factor influencing polymer aggregation. When statistical copolymers are used, the presence of multiple groups on the backbone can result in interactions with several particles leading to flocculation. In the case of di- and multi-block polymers, the functional block should be kept relatively short (several units) to avoid flocculation. The introduction of several attachment points on a single chain can reduce the packing density. Using this multi-interaction approach, many different types of functionalities have been used, such as dopamine [29], carboxylic acid [30] and trimethyl silane [31], yielding stable nanoparticles. The acid groups, not used for IONP stabilization, were exploited for the attachment of Cy5.5 dye [11] and small-molecule drugs such as doxorubicin [32].

Micelle and liposome encapsulation. Stabilization of IONPs using micelles and liposomes has also been described in the literature. Liposomes are large, closed, tertiary structures consisting of phospholipid bilayers with sizes in the range 100-5,000 $\mathrm{nm}$. IONPs can be stabilized by hydrophobic interactions involving the phospholipids. In addition, a single liposome can encapsulate several IONPs within its hydrophobic layer. 

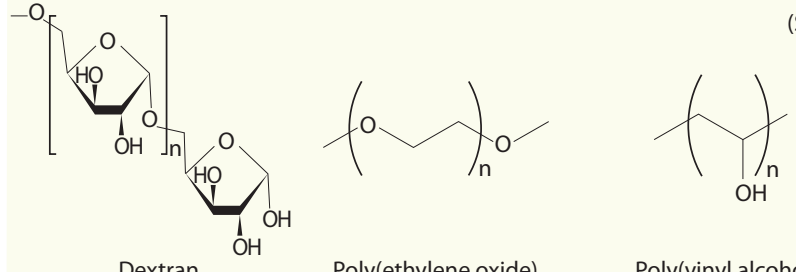

Dextran

Poly(ethylene oxide)
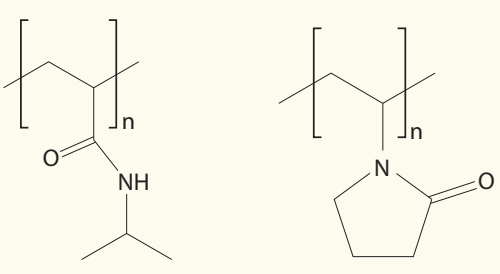

Poly(vinyl alcohol)

Poly(N-isopropylacrylamide)

Poly(vinylpyrrolidone)

Poly(oligoethylene oxide)
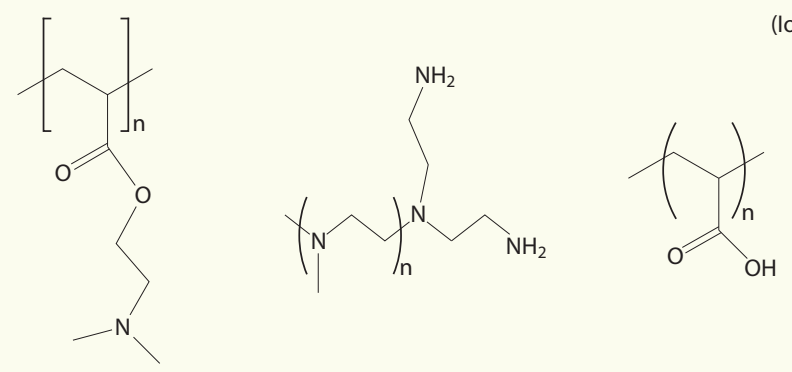

Poly(N,N-dimethyl ethylamino acrylate)

Poly(imine)

Poly(acrylic acid)

(lonic)

Figure 4. Polymers used for the stabilization of IONPS.
(Steric)

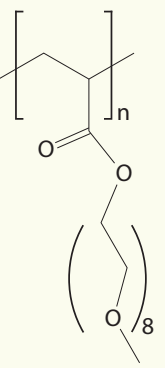

\section{Toxicity of IONPs}

IONPs generally show low toxicity as they degrade into $\mathrm{Fe}^{2+}$ and $\mathrm{Fe}^{3+}$ [40]. However, magnetite and maghemite nanoparticles show different toxicities [41]. Magnetite has been found to display cytotoxicity as it can degrade via the Fenton reaction: $\mathrm{H}_{2} \mathrm{O}_{2}+\mathrm{Fe}^{2+} \rightarrow \mathrm{Fe}^{3+}+\mathrm{HO}^{-}+\mathrm{HO} \bullet$ generating a reactive free radical. In addition, an excess of iron in the body is undesirable and is associated with a number of diseases; for example, in the brain, iron can induce neurodegenerative disorders (Alzheimer's and Parkinson's diseases) [42]. However, in vivo studies have shown that IONPs are relatively safe as they do not accumulate in the vital organs and are rapidly eliminated from the body. The presence of a polymer coating, such as PEG, can also mediate IONP toxicity, as demonstrated for human fibroblasts $[41,43]$.

\section{Relationship between physicochemical properties of polymers and blood circulation half-life}

Nanocarriers and MRI contrast agents need to have long blood circulation times and must evade the reticuloendothelial system to accumulate in target tissues [44]. The physicochemical properties of IONPS, such as size, charge, polymer density and morphology, play a crucial role in determining their blood half-life [45].

Several studies have described the effect of size on the stability of IONPs in vivo. Particles with sizes in the range $10-100 \mathrm{~nm}$ are optimal for long circulation times in vivo. Large particles $(>200 \mathrm{~nm})$ are readily sequestered by the phacocytic cells in the spleen [46] or by the macrophage cells present in blood, while very small IONPs $(<10 \mathrm{~nm})$ are rapidly removed by the renal clearance process [38]. Perrault and co-workers [47] examined the effects of core (hard part) size and the polymer (PEG, soft part) chain length on the biodistribution of IONPs, and found that the blood half-life decreased as the core diameter increased; an increase in PEG molecular weight was found to cause a significant increase in circulation time.

Shape is a factor in determining the stability and biodistribution of nanoparticles [48]. Indeed, Decuzzi and Ferrari [49] established via simulation that oblate spheroid nanoparticles show a longer circulation time than spherical nanoparticles. Recently, this theoretical result has been validated by several studies. For instance, Muro et al. [50] compared the biostability of spheres of various diameters versus elliptical discs, showing that elliptical discs have longer circulation times. A similar result has been reported for spherical gold nanoparticles and nanorods [51]; cell uptake was found to be three times higher for spherical gold nanoparticles. Spherical particles need to have sizes smaller than $200 \mathrm{~nm}$ to pass through the spleen; in contrast, elliptical nanoparticles (discs) 
with sizes greater than $1,000 \mathrm{~nm}$ can pass through [49]. This unexpected result has been explained by auto-organization (alignment or tumbling) of non-spherical nanoparticles under the influence of flow [49]. However, there is still much work needed to fully understand the role of nanoparticle shape on both their biodistribution and cell uptake.

The texture of hybrid nanoparticles, including the mechanical properties of the polymer coating, has a major role in determining protein adsorption. Ideally, the polymer layer is at the interface of the IONP surface interacting with the biological medium, mediating protein adsorption [35]. Charged polymers can cause colloidal instability in biological fluids, such as plasma, through protein adsorption. Investigations into the effect of nanoparticle surface charge (i.e. zetapotential) on stability in biological media have shown that an increase in plasma protein adsorption occurs when the surface charge density is increased. The level of protein adsorption, for any given nanoparticle charge density, will be dependent on the protein structure. Negatively charged nanoparticles bind with proteins having isoelectric points greater than 5.5, such as IgG. In contrast, positively charged particles bind to proteins having isoelectric points less than 5.5, such as albumin [35].

Hydrophobic polymers bound to nanoparticle surfaces can also interact with biological media through specific hydrophobic interactions. The amount and nature of protein adsorption to hybrid nanoparticles is strongly influenced by the hydrophobicity of the polymer [52]. Nanoparticles with hydrophobic surfaces exhibit more susceptibility to opsonization, a process whereby the body tags particles for ingestion and destruction by phagocytes, than more hydrophilic nanoparticles. Cedervall et al. [53] quantified both the nature and the amount of protein adsorbed to nanoparticles with varying hydrophobicity, establishing that hydrophilic particles present a relatively strongly antifouling surface, binding only to albumin; in contrast, hydrophobic surfaces were found to bind to several proteins, including albumin, IgG, apoliproteins and fibrinogen.

One of the most efficient polymers employed in preventing protein binding on surfaces is PEG, which has been used to limit interactions between proteins and nanoparticles surfaces [54]. There are two significant factors that are known to influence the efficiency of PEGamelioration of protein binding to surfaces: molecular weight and grafting density. Increasing the molecular weight or density of PEG chains causes a decrease in protein adsorption [55]. PEGylated nanoparticles show a lower cell uptake rate by macrophages (polymorphonuclear cells), resulting in an increase in the blood circulation time [31,33,36].

Biocirculation of nanoparticles can thus be adversely affected by specific protein binding. However, in some cases, the presentation of specific proteins on nanoparticle surfaces can be useful for aiding passage through biological barriers; the presence of apolipoprotein on nanoparticles, for instance, can facilitate passage through the bloodbrain barrier [56].

\section{Functionalization strategies}

Passive targeting. Long blood-circulation time is known to be beneficial for promoting the accumulation of nanoparticles smaller than $500 \mathrm{~nm}$ in tumors and at inflammatory and infection sites due to the enhanced permeability and retention (EPR) effect, offering a mechanism for passive targeting. EPR is caused by increased permeability of the vascular system close to tumor sites and inefficient lymphatic drainage. Passive targeting has been demonstrated for nanoparticles with sizes ranging from 10 to $500 \mathrm{~nm}$ [57]. Perrault et al. [47] studied the effect of nanoparticle size $(20-100 \mathrm{~nm})$ on accumulation at tumor sites and found that particle accumulation $(40-100 \mathrm{~nm})$ depends only on the blood residence half-life and is independent of nanoparticle size; in contrast, for smaller particles (around $20 \mathrm{~nm}$ ), the accumulation depends on both factors. However, small particles have a relatively short residence time at the tumor site when compared with larger particles $(>40 \mathrm{~nm})$. Small nanoparticles therefore arrive rapidly at the tumor site, but also have a short residence time, whereas larger nanoparticles take longer to reach the tumor sites, but reside for longer in the blood.
The residence time is a key parameter for therapeutic efficiency, as the nanoparticles are initially transported by the blood, followed by passive diffusion from the blood vessel to the tumor periphery. The distribution (and therefore efficiency) of the nanoparticles depends on both size and residence time in the vicinity of the tumor [47].

A different passive targeting technique has been developed for IONPs using reticuloendothelial system clearance to facilitate imaging of specific organs, such as the spleen or liver. This targeting method relies on the rapid uptake of IONPs by macrophages. The first commercial contrast agents (Feridex IV) exploited this targeting method to image the presence of infected tissue in the liver [58]. The rapid uptake of IONPs by Kupffer cells in the liver allows differentiation of healthy tissues from tumor cells using MRI. However, this macrophage-assisted targeting method is limited to specific organs such as the liver and spleen (both rich in macrophages) or inflamed tissues.

Active targeting. Improved MRI resolution can also be envisaged by adopting an active targeting IONP strategy using biological recognition events. A range of targeting compounds have been described in the literature, including peptides (RGD, NGR) [22], proteins (monoclonal antibodies) [59], aptamers [60], carbohydrates [61] and small molecules (folic acid) [24]. This active-targeting approach has the potential to reduce the IONP concentration required for clinical use whilst still maintaining sufficient MRI resolution.

The attachment of targeting functionality to IONPs has been achieved using a number of synthetic strategies, including carboxylic acid-amine reactions [62], click chemistry (i.e. CuAAC) [63], pyridyl disulfide-thiol exchange and thiol-ene reactions [64]. The CuAAC approach suits polymers synthesized via atom transfer radical polymerization (ATRP), while thiol-ene or pyridyl-disulfide exchange reactions are more appropriate for the functionalization of polymers obtained using reversible addition-fragmentation transfer polymerization (RAFT) [65]. RAFT polymers can be converted easily to terminal thiol [66], and are amenable to subsequent diverse reactions, such as thiol-ene and pyridyl-disulfide exchange reactions [66].

The accessibility of the targeting groups on IONP surfaces is important, as shown by Martin et al. [61], who found that differences in accessibility induced different biological responses. Targeting moieties (i.e. lactose) fixed on a dendrimer presented improved exposure and binding (by 1-2 orders of magnitude) compared to monofunctional lactose terminated polymers. An enhanced availability of dendritic molecules on the surface prevents interment of the lactose within the polymer coating. In addition, the fixed presentation of the targeting moieties by the dendritic structure enhances binding via a concentration effect known as multivalency. The study by Martin et al. [61] proves that the binding affinity of carbohydrate ligands when presented on polymer surfaces can be significantly enhanced through the use of dendritic scaffolds. However, the importance of multivalent interactions depends to some extent on the specific biological binding event, and therefore some caution should be exercised in extending the conclusions from the study of Martin et al. to all targeting interactions.

Combining a targeting agent with a fluorescence label on nanoparticles produces multi-functional and multi-modal nanoparticles that have both a 'tracking signal' and a high affinity for specific targeted cell types. For example, Wang et al. [67] modified IONPs with a poly(amidoamine) dendrimer, following by the attachment of folic acid, producing IONPs that were readily taken up by $\mathrm{KB}$ tumor cells with folic acid receptor expression (KB-HFAR).

\section{Applications}

IONPs can be employed in many applications, and in medicine are primarily applied as contrast agents in MRI and in drug and gene delivery [68]. IONPs are also used in hyperthermia therapy, a treatment by which body tissue is exposed to (locally) high temperatures thereby damaging and killing cancer cells. The fundamental magnetic properties 


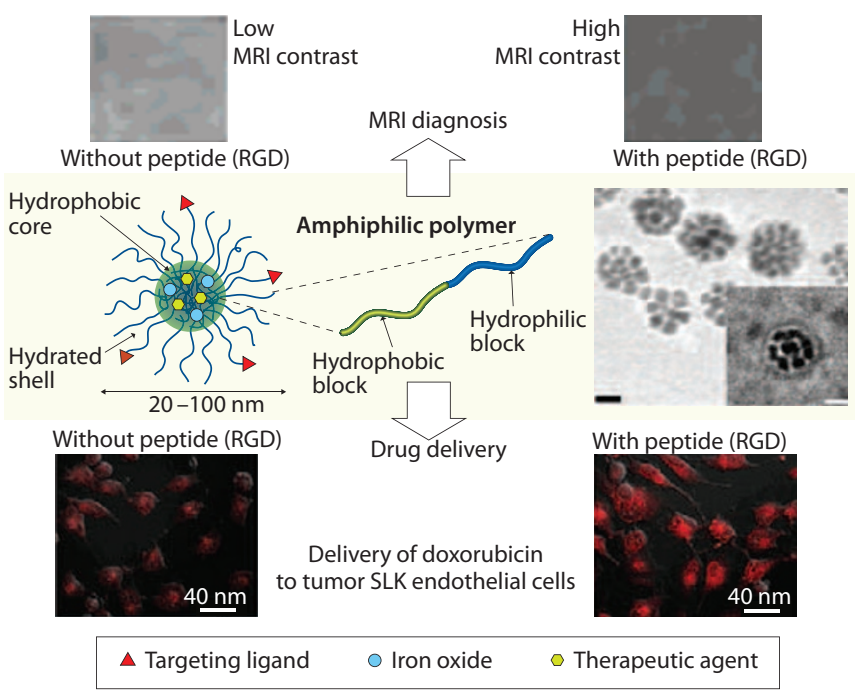

Figure 5. Example of multi-functional IONPs used for drug delivery and as MRI contrast agents. (Top) $T_{2}$-weighted MR images (4.7T, spin echo acquisition, $T_{\mathrm{R}}=6000 \mathrm{~ms}, T_{\mathrm{E}}=90 \mathrm{~ms}$ ) of Ste20-like kinase (SLK) cells, untreated (left) and treated (right) with 16\% CRGD-doxorubicin-IONPs micelles at an iron concentration of $25 \mu \mathrm{g} \mathrm{mL}^{-1}$. (Middle) Schematic representation of IONPs stabilized using an amphiphilic polymer (poly(ethylene glycol)-block-poly(D,L-lactide)). The hydrophobic core encapsulates doxorubicin as a therapeutic agent, and the particles are decorated with a targeting agent (c-RGD peptide). The transmission electronic microscopy (TEM) images (cryo-TEM inset) show doxorubicin-IONP-loaded polymeric micelles. (Bottom) Fluorescence microscopy of cell uptake of IONPs in SLK cells with and without c-RGD peptide using doxorubicin as a fluorescence label. Adapted from Ref. 34 (๔ 2005 ACS)

of IONPs are pivotal for all these applications. The use of IONPs in MRI and drug and gene delivery is described in more detail below.

\section{Magnetic resonance imaging}

MRI was developed in 1973 and has since become one of the most powerful non-invasive techniques used in clinical medicine, allowing the internal structure and function of the body to be visualized [69]. MRI utilizes the magnetic spins of hydrogen nuclei aligned by a powerful external magnetic field. A radiofrequency pulse disturbs the spin alignment from equilibrium, and the spin relaxation back to equilibrium is monitored at high temporal resolution. Two independent relaxation processes occur in MRI: longitudinal relaxation denoted $T_{1}$, and transverse relaxation denoted $T_{2}$. These two relaxation processes can be monitored independently to generate different MR images, such as $T_{1^{-}}, T_{2^{-}}$and $T_{2}{ }^{*}$-weighted images. Local fluctuations in proton spin density, mainly attributed to water molecules and caused by variations in the biological environment, affect the relaxation responses from which images can be constructed.

Improvement in MRI contrast can be effected using magnetic nanoparticles to shorten the relaxation times $\left(T_{1}\right.$ and $\left.T_{2}\right)$. IONPs influence the $T_{2}$ time for water, while gadolinium-modified nanoparticles (another type of contrast agent for MRI) influence the longitudinal relaxation $\left(T_{1}\right)$ [70]. These two relaxation modes yield different MR images.

The efficacy of a nanoparticle contrast agent can be characterized by measuring the relaxivity, $R_{1}$ and $R_{2}$, of water protons surrounding the nanoparticle. The relaxivity is inversely proportional to the individual measured relaxation rates $\left(R_{1}=1 / T_{1}, R_{2}=1 / T_{2}\right)$ over a range of contrast concentrations. The magnetic properties of the iron cores (magnetic saturation) influence the values of both $R_{1}$ and $R_{2}$. A higher $R_{2} / R_{1}$ ratio improves the contrast. Iron-oxide contrast agents such as Resovist and Endorem have relaxivities in the range of $150-160 \mathrm{~s}^{-1}$ per millimole. The quality of a contrast agent (in vivo) depends on the IONP core, and also on its ability to evade the reticuloendothelial system clearance mechanism, its stability in biological fluids and its ability to concentrate at the target area. The polymer coating plays a significant role in tuning the properties of IONPs thereby mediating the interface between the IONP surface and the biological medium. The ways in which the design of the polymer layer can affect the MRI properties of the hybrid nanoparticles are discussed below.

The nature of the ligand, the linker between IONPs and polymer, can affect the organization of magnetic spins in the IONPs, resulting in modified magnetic properties [71]. Daou and co-workers [72] compared the effect of two different ligand types, phosphonate and carboxylate, on the magnetic properties of IONPs, showing that phosphonate ligands yielded superior magnetic properties due to the absence of spin canting in the IONP layer close to the interface.

The chain length of polymers may also have an effect on the relaxivity properties of the nanoparticles. The magnitude of MRI relaxivity is dependent on the number of water molecules disturbed by the magnetic field generated by the IONPs, which may be influenced by the thickness and nature of the polymer layer. Laconte et al. [73] reported a decrease in $R_{2}$ with increasing chain length (or molecular weight) of the polymer coating.

Duan et al. [74] examined the effect of polymer hydrophobicity on the magnetic properties (relaxivities) of IONPs, and found that the hydrophobic polymers diminished the relaxivity $\left(R_{2}\right)$ behavior. In contrast, IONPs with a core size of approximately $10 \mathrm{~nm}$ coated with PEI, a hydrophilic charged polymer, presented significantly enhanced relaxivity. A similar result was also observed for IONPs with a core size greater than $30 \mathrm{~nm}$, although the difference was less significant.

\section{Drug and gene delivery}

Several reports have been published on the use of IONPs as nanocarriers for drug and gene delivery [75]. The presence of a magnetic core offers the promise of targeting specific organs within the body [76]. Magnetic focusing can be exploited to concentrate the IONPs in the desired area such that the accumulation of IONPs can exaggerate the EPR effect. In addition, the iron-oxide core can be engineered to liberate toxic organic compounds by the introduction of platinum inside the IONP cores [77].

The polymer coating can also now be exploited, not just for stabilizing the IONPs in biological media, but also as a scaffold (reservoir) for the drug or gene cargo. The loading and release of bioactive materials from the polymer coating then becomes a significant parameter dictating the efficiency of IONPs as nano-carriers. Therapeutics can be conjugated to the polymer chains using a number of alternative approaches: covalent coupling, charge complexation, hydrogen bonding or hydrophobic/hydrophilic interactions. The therapeutic agent can also be attached directly onto the IONP surface. Gendeli and coworkers [78] covalently attached an anti-cancer drug (amptothecin) to USPIOs (core diameter, 9-10 nm; hydrodynamic diameter, $52 \mathrm{~nm}$ ) coated with polyvinylalcohol/polyvinylamine using a biodegradable linkage. The magnetic properties of the nanoparticles were exploited to enhance cell uptake.

Yu et al. [32] developed IONPs bearing (poly(OEG-A)) that are able to conjugate doxorubicin via a $\mathrm{pH}$-sensitive bond. Doxorubicin release could be mediated by manipulating $\mathrm{pH}$, whilst the magnetic properties of the IONPs were completely preserved. Hydrophobic interactions between polymers and drugs have also been exploited, as demonstrated by Nasongkia and co-workers [34], who loaded doxorubicin into hydrophobic cores stabilized with a hydrophilic shell and decorated with targeting agents (RGD peptide). Drug release was controlled by degradation of the poly(D,L-lactide) cores and $\mathrm{pH}$ control. The presence of a targeting agent was shown to improve the delivery of doxorubicin to specific cells (Figure 5).

Gong et al. [79] created microcapsules of around $50 \mu \mathrm{m}$ in size using a microfluidic process, yielding shells comprised of IONPs embedded in a dextran layer surrounding a core loaded with aspirin. On application of an alternating magnetic field, the shell was deformed, releasing the aspirin. 
Liu et al. [80] exploited the ability of IONPs to generate heat when they are subjected to a high-frequency oscillating magnetic field. In their work, IONPs were coated with a thermo-responsive anti-fouling polymer (pluronic PEG- $b$-PEO- $b$-PEG), which was cross-linked using gelatin. Drugs were encapsulated in the polymer shell using hydrophobic interactions and then released by induced heating stimulated by the magnetic field.

DNA- and siRNA-based therapies have shown great promise for the treatment of disease [81]. However, realizing the potential in practice is not straightforward as the nucleotide therapeutics need to be protected from enzymatic degradation during circulation. Various carriers have been proposed, including viruses, polymers nanoparticles, bioconjugates and organic/ inorganic particles [82]. The development of nano-carriers for siRNA has received particular attention [82]. In 2009, several publications described the use of organic/inorganic nanoparticles (e.g. gold and IONPs) for siRNA delivery. The application of hybrid metal nanoparticles to gene therapy has some advantages over other delivery methods. Firstly, fixed shapes and topologies can be engineered, and secondly, the presence of inorganic cores with magnetic (e.g. IONPs) or optical (gold nanoparticles) properties permits tracking of hybrid nanoparticles in vitro.

The conjugation of oligonucleotides to carrier nanoparticles can be achieved using electrostatic interactions with cationic polymers or by covalent attachment either to the polymer [83] or directly to the nanoparticle [84]. A number of cationic polymers (e.g. poly(ethylene imine) [85] and poly(dimethyaminoethyl (meth)acrylate) [86]) have been grafted onto IONPs and used for conjugation with siRNA via electrostatic interactions. Pan et al. [85] developed PEI-coated IONPs for the encapsulation of genes and demonstrated successful in vitro delivery. Several other studies have also demonstrated the feasibility of using hybrid nanoparticles for the delivery of DNA or siRNA in vitro. Magnetic cores have been exploited for enhanced transfection under a magnetic field (magnetofection) [37]. Kamau et al. [86] functionalized IONPs with PEI for gene delivery applications and studied their transfection efficiency under an applied magnetic field in vitro. On exposure to permanent and oscillating magnetic fields, the in vitro transfection efficiency was 40 times higher than in the absence of a magnetic field. However, the presence of cationic polymer on the surface of these nanoparticles may cause severe problems if the nanoparticles were to be used in vivo, as significant protein adsorption would occur in biological media. An enhanced design of IONPs for gene delivery has been proposed by the Centre for Advanced Macromolecular Design based on a mixed polymer layer approach using both charged (cationic poly(dimethylaminoethyl acrylate)) and neutral (poly(OEG-A)) polymers co-grafted onto IONPs to yield uncharged particles (Figure 6) [87]. The presence of positive charges within the polymer layer facilitates complexation with siRNA, and the siRNA/ particle complexes display excellent stability in fetal bovine serum and have an effect on transfection in vitro, both with and without a magnetic field.

Medarova et al. [83] used a different strategy to deliver siRNA with IONPs based on the use of multimodal IONPs. First, IONPs were coated with a dextran polymer layer bearing amine groups. A peptide (myristoylated polyarginine peptide) serving as a membrane translocation module and an optical label (Cy5.5, used for nearinfrared in vivo optical imaging) were then fixed on the dextran by traditional coupling chemistry. The residual amines not used for the coupling process were then accessed and used for the attachment of siRNA. This platform allows the simultaneous delivery of siRNA and particle tracking in vivo through a combination of near-infrared optical imaging and MRI. Lee et al. [84] attached siRNA directly onto IONP surfaces using disulfide bonds. To enhance the solution stability of these nanoparticles in vivo, a thiol-functionalized PEG was also grafted to the nanoparticle surface. Targeting and enhanced tracking were achieved using a targeting agent (RGD peptide) and a label (Cy5.5 for nearinfrared in vivo optical imaging).

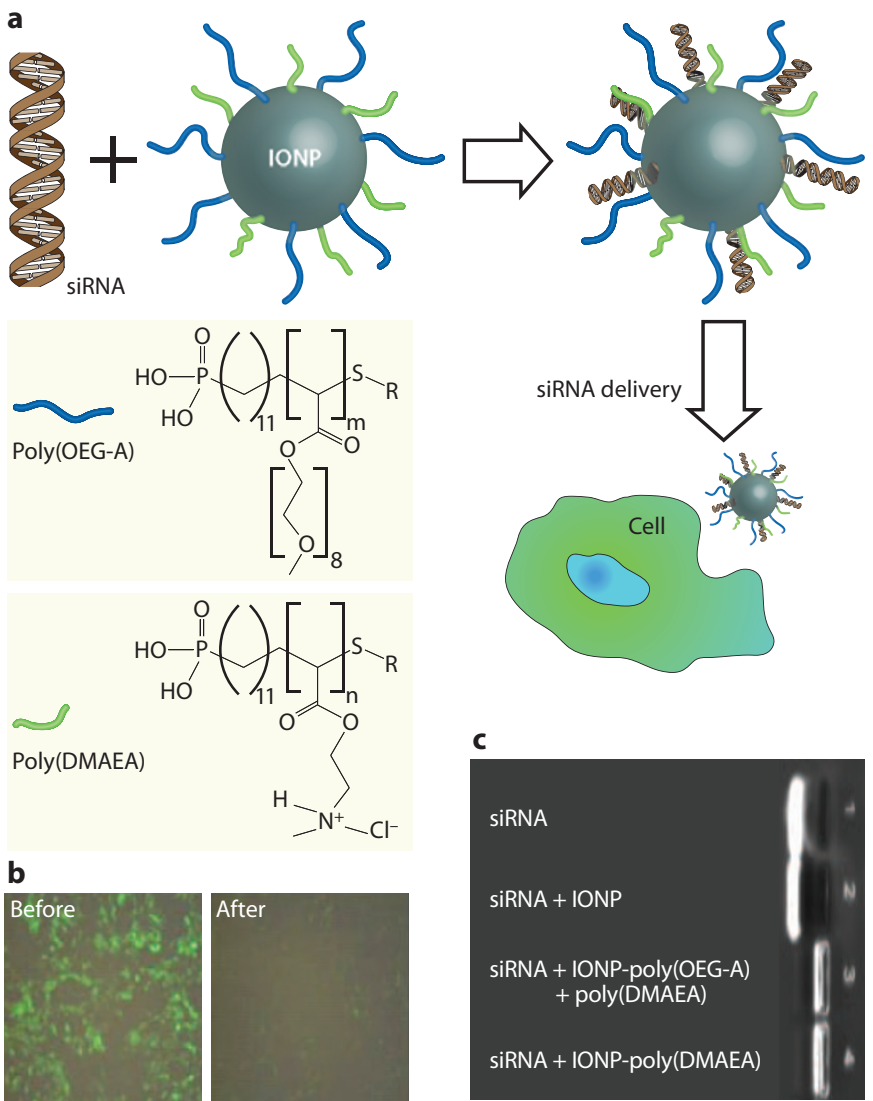

Figure 6. siRNA delivery using IONPs. (a) siRNA encapsulation using IONPs bearing poly(oligoethylene glycol) (poly(OEG-A)) and poly(dimethylaminoethyl acrylate) (poly(DMAEA)) hydrochloride. (b) Fluorescence microscopy of SHEP/GFP cells before and after addition of IONPs (24h) under a magnetic field. (c) Agarose gel of siRNA complexed with IONPS.

\section{Conclusion}

This review presented an overview of the synthesis, surface modification and use of IONPs for biological applications. Extremely versatile and multi-functional nanoparticles can be made by combining the favorable properties of the metal cores with the functionality of polymer shells. The polymers can confer many excellent properties to the IONPs, including stabilization in biological fluids, targeting and drug and gene portability and delivery, and these benefits can be combined with the MRI properties of the iron cores.

\section{Acknowledgments}

This work was supported by an Australian Research Council Discovery grant (DP1092640) (T. P. Davis, C. Boyer), and a Federation Fellowship (T. P. Davis).

\section{References}

[1] M. De, P. S. Ghosh, V. M. Rotello, Adv. Mater. 20, 4225 (2008).

[2] Y.-W. Jun, J.-H. Lee, J. Cheon, Angew. Chem. Int. Ed. 47, 5122 (2008).

[3] S. Laurent et al., Chem. Rev. 108, 2064 (2008).

[4] J. Gao, H. Gu, B. Xu, Acc. Chem. Res. 42, 1097 (2009).

[5] S. Peng, C. Wang, J. Xie, S. Sun, J. Am. Chem. Soc. 128, 10676 (2006).

[6] S. Sun et al., J. Am. Chem. Soc. 126, 273 (2004).

[7] W. Y. Teoh et al., NSTI Nanotech 2007 4, 187 (2007).

[8] D. Li et al., Chem. Mater. 18, 6403 (2006).

[9] J.-W. Jun et al., J. Am. Chem. Soc. 127, 5732 (2005).

[10] A. K. Gupta, M. Gupta, Biomater. 26, 3995 (2005).

[11] H. Lee et al., J. Am. Chem. Soc. 129, 12739 (2007). 
[12] R. De Palma et al., Chem. Mater. 19, 1821 (2007).

[13] K. El-Boubbou, C. Gruden, X. Huang, J. Am. Chem. Soc. 129, 13392 (2007).

[14] Y. Sahoo et al., Langmuir 17, 7907 (2001).

[15] L. Wang et al., J. Am. Chem. Soc. 128, 13358 (2006).

[16] K. Cheng, S. Peng, C. Xu, S. Sun, J. Am. Chem. Soc. 131, 10637 (2009)

[17] M. D. Shultz, J. U. Reveles, S. N. Khanna, E. E. Carpenter, J. Am. Chem. Soc. 129, 2482 (2007).

[18] M. Lattuada, T. A. Hatton, Langmuir 23, 2158 (2007).

[19] J. Tian, Y. K. Feng, Y. S. Xu, Macromol. Res. 14, 209 (2006).

[20] L. Wang, K. G. Neoh, E. T. Kang, B. Shuter, S.-C. Wang, Adv. Funct. Mater. 19, 2615 (2009).

[21] C. Flesch et al., Macromol. Rapid Commun. 26, 1494 (2005).

[22] C. Boyer et al., J. Mater. Chem. 19, 111 (2009).

[23] R. Narain, M. Gonzales, A. S. Hoffman, P. S. Stayton, K. M. Krishnan, Langmuir 23, 6299 (2007).

[24] G. Huang et al., J. Mater. Chem. 19, 6367 (2009).

[25] N. Kohler, G. E. Fryxell, M. Zhang, J. Am. Chem. Soc. 126, 7206 (2004).

[26] E. Amstad et al., Small 5, 1334 (2009).

[27] C. D. Hein, X.-M. Liu, D. Wang, Pharm. Res. 25, 2216 (2008)

[28] M. A. White, J. A. Johnson, J. T. Koberstein, N. J. Turro, J. Am. Chem. Soc. 128, 11356 (2006)

[29] M. I. Shukoor et al., Chem. Mater. 20, 3567 (2008).

[30] F. Zhang, C.-C. Wang, Langmuir 25, 8255 (2009).

[31] H. Lee et al., J. Am. Chem. Soc. 128, 7383 (2006).

[32] M. K. Yu et al., Angew. Chem. Int. Ed. 47, 5362 (2008).

[33] J. Park et al., J. Mater. Chem. 19, 6412 (2009).

[34] N. Nasongkla et al., Nano Lett. 6, 2427 (2006).

[35] P. Aggarwala, J. B. Halla, C. B. McLelanda, M. A. Dobrovolskaia, S. E. McNeila, Adv. Drug Deliv. Rev. 61, 428 (2009).

[36] G. Prencipe et al., J. Am. Chem. Soc. 131, 4783 (2009).

[37] X. Wang, L. Zhou, Y. Ma, X. Li, H. Gu, Nano Res. 2, 365 (2009).

[38] H. S. Choi et al., Nature Biotechnol. 25, 1165 (2007).

[39] O. Mykhaylyk, Y. S. Antequera, D. Vlaskou, C. Plank, Nature Protoc. 2, 2391 (2007)

[40] A. Nel, T. Xia, L. Mädler, N. Li, Science 311, 622 (2006).

[41] A. K. Gupta, A. S. G. Curtis, J. Mat. Sci.: Mat. Med. 15, 493 (2004).

[42] P. M. Doraiswamy, A. E. Finefrock, Lancet Neurol. 3, 431 (2004).

[43] Y. Wang et al., Adv. Funct. Mater. 18, 308 (2008).

[44] S. M. Moghimi, A. C. Hunter, J. C. Murray, Pharmacol. Rev. 53, 283 (2001).

[45] F. Alexis, E. Pridgen, L. K. Molnar, O. C. Farokhzad, Mol. Pharmaceutics 5, 505 (2008).

[46] Y. Tabata, Y. Ikada, Adv. Polym. Sci. 94, 107 (1990).

[47] S. D. Perrault, C. Walkey, T. Jennings, H. C. Fischer, W. C. W. Chan, Nano Lett. 9, 1909 (2009).
[48] S. Mitragotri, Pharm. Res. 26, 232 (2009).

[49] P. Decuzzi, M. Ferrari, Biomater. 27, 5307 (2006).

[50] S. Muro et al., Mol. Therap. 16, 1450 (2008).

[51] B. D. Chithrani, A. A. Ghazani, W. C. W. Chan, Nano Lett. 6, 662 (2006).

[52] D. E. Owens, N. A. Peppas, Int. J. Pharm. 307, 93 (2006).

[53] T. Cedervall et al., Angew. Chem. Int. Ed. 46, 5754 (2007).

[54] H. R. Kim et al., Electrophoresis 28, 2252 (2007).

[55] R. Gref et al., Colloids Surf., B 18, 301 (2000).

[56] T. M. Göppert, R. H. Müller, J. Drug Target. 13, 179 (2005).

[57] D. Peer et al., Nature Nanotech. 2, 751 (2007).

[58] D. D. Stark et al., Radiology 168, 297 (1988).

[59] P. Carter, Nature Rev. Cancer 1, 118 (2001).

[60] F. Gu et al., PNAS 105, 2586 (2008).

[61] A. L. Martin, B. Li, E. R. Gillies, J. Am. Chem. Soc. 131, 734 (2009).

[62] X. Shi, T. P. Thomas, L. A. Myc, A. Kotlyar, J. R. Baker, Phys. Chem. Chem. Phys. 9, 5712 (2007)

[63] R. A. Evans, Aust. J. Chem. 60, 384 (2007)

[64] K. L. Killops, L. M. Campos, C. J. Hawker, J. Am. Chem. Soc. 130, 5062 (2008).

[65] C. Boyer et al., Chem. Rev. 109, 5402 (2009).

[66] C. Boyer, V. Bulmus, T. P. Davis, Macromol. Rapid Commun. 30, 493 (2009).

[67] S. H. Wang et al., Adv. Funct. Mater. 17, 3043 (2007).

[68] M. Namdeo et al., J. Nanosci. Nanotech. 8, 3247 (2008).

[69] K. N. Raymond, V. C. Pierre, Bioconjugate Chem. 16, 3 (2005).

[70] Y.-X. Wang, S. M. Hussain, G. P. Krestin, Eur. Radiol. 11, 2319 (2001).

[71] C. R. Vestal, Z. J. Zhang, J. Am. Chem. Soc. 125, 9828 (2003).

[72] T. J. Daou et al., Chem. Mater. 20, 5869 (2008).

[73] L. E. W. LaConte et al., J. Magn. Res. Imag. 26, 1634 (2007).

[74] H. Duan et al., J. Phys. Chem. C 112, 8127 (2008).

[75] J. Dobson, Gene Therapy 13, 283 (2006).

[76] L. Zhang et al., Clin. Pharmacol. Ther. 83, 761 (2008).

[77] J. Gao et al., J. Am. Chem. Soc. 130, 11828 (2008).

[78] F. Cengelli et al., ChemMedChem 4, 988 (2009).

[79] X. Gong, S. Peng, W. Wen, P. Sheng, W. Li, Adv. Funct. Mater. 19, 292 (2009)

[80] T.-Y. Liu, K.-H. Liu, D.-M. Liu, S.-Y. Chen, I.-W. Chen, Adv. Funct. Mater. 19, 616 (2009)

[81] D. S. Shewach, R. D. Kuchta, Chem. Rev. 109, 2859 (2009).

[82] A. Akinc et al., Nature Biotech. 26, 561 (2008).

[83] Z. Medarova, W. Pham, C. Farrar, V. Petkova, A. Moore, Nature Med. 13, 372 (2007)

[84] J.-H. Lee et al., Angew. Chem. Int. Ed. 48, 4174 (2009).

[85] B. Pan et al., Cancer Res. 67, 8156 (2007).

[86] S. W. Kamau et al., Nucl. Acids Res. 34, e40 (2006).

[87] C. Boyer et al., J. Mater. Chem. 20, 255 (2010).

\section{Author profiles}

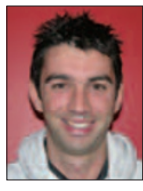

\section{Cyrille Boyer}

Cyrille Boyer received his PhD in polymer chemistry in 2005 from the University of Montpellier II in France. Since October 2006, he has worked as a senior research fellow in the Centre for Advanced Macromolecular Design. His research interests mainly cover the preparation of hybrid organic-inorganic nanoparticles using controlled radical polymerization and well-defined protein-polymer conjugates.

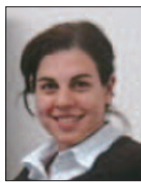

\section{Volga Bulmus}

Volga Bulmus is a senior lecturer at the School of Biotechnology and Biomolecular Sciences, University of New South Wales, and an adjunct member of the Centre for Advanced Macromolecular Design working on the development of advanced polymers for biotechnology and biomedical applications. Her research interests include the design, synthesis and evaluation of well-defined polymeric systems for nanobiotechnology and drug delivery applications.

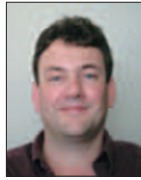

\section{Thomas P. Davis}

Tom Davis has been an academic at the University of New South Wales since 1993. He is founding director of the Centre for Advanced Macromolecular Design, and has initiated and led research programs on RAFT polymerization (kinetics, mechanism and complex architectures), mass spectrometry of polymers, honeycomb films from breath figures, bioconjugation, glycopolymers and nanoparticles.

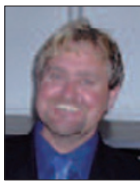

\section{Michael R. Whittaker}

Michael Whittaker obtained his PhD in 2000 from the University of Queensland, Australia. He has since spent time in both industry and academia. In 2008, he joined the Centre for Advanced Macromolecular Design as center research manager and senior research fellow. His current research interests focus on the synthesis of 'smart' hybrid inorganic/organic nano-materials.

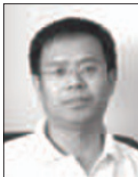

\section{Jingquan Liu}

Jingquan Liu obtained his master and PhD degrees at the University of New South Wales in 1999 and 2004. After two years as a postdoctoral fellow at the University of Technology Sydney and with the CSIRO, he returned to the University of New South Wales as a Vice-Chancellor's research fellow. His research interests focus on the synthesis of various bio- and nano-hybrids of versatile biodegradable and functional polymeric architectures. 